\title{
Qualitative Analysis of Modified Hand Test
}

\author{
Fayyaz Ahmed Anjum ${ }^{1 *}$, Dr Iffat Batool ${ }^{2}$
}

\section{ABSTRACT}

Qualitative analysis of modified Hand Test was carried out on 500 participants further bifurcated into 350 normal, 50 maladjusted, 50 neurotic and 50 psychotic participants. Their ages ranged from 11 years to 90 years with mean age of 34.44 SD (17.34). The qualitative analysis based up on seventeen categories which includes ambivalent, automatic phrase, cylindrical, denial, emotion, gross, hiding, immature, impotent, inanimate, movement, oral, and perplexity, sensual, sexual and original. Original Purposive sampling technique was used. Modified Hand test with four new adapted stimulus was administered in accordance with described instructions by its author. Testing the limit procedure was applied only for psychotic group. Post-test inquiry was held to clarify certain responses. The results of the study depicted interesting features which differentiates four groups. E.g. normal group did not produce any sexual, hiding, repetition responses. More number of repetitive responses were found in mal adjective and neurotic groups. Certain new areas were also explored like introjections produced by psychotic group. Need for altruism by doctors sub group of normal population. Direction by teachers. Interesting results are expected with other different sample and are likely to provide insight in order to understand human behavior in tradition of idiosyncratic approach.

Keywords: Qualitative Analysis, Modified Hand Test

Hand test was developed by Edwin E Wagner in 1962 and new norms for children and adolescents were introduced in 1983. Hand test has gained reputation of projective and diagnostic measure and became popular amongst clinicians and researchers because it has the capacity to measure various aspects of Human Personality and Behavior. Despite being projective test, it offers objectivity in terms of well-defined quantitative scoring systems and on this aspect it enjoys leading role amongst other projective techniques, because subjectivity in terms of interpreting responses is a major criticism on projective techniques (Anastasi, 1997), (Maha Nazir, 2008). In addition to quantitative scoring categories the author of the Test had also

\footnotetext{
${ }^{1}$ PhD Scholar, Department of Psychology, Government College University, Lahore, Pakistan

${ }^{2}$ Faculty, Department of Psychology, Government College University, Lahore, Pakistan

*Responding Author
}

Received: March 2, 2017; Revision Received: March 26, 2017; Accepted: March 29, 2017

(C) 2017 Anjum F, I Batool; licensee IJIP. This is an Open Access Research distributed under the terms of the Creative Commons Attribution License (www.creativecommons.org/licenses/by/2.0), which permits unrestricted use, distribution, and reproduction in any Medium, provided the original work is properly cited. 


\section{Qualitative Analysis of Modified Hand Test}

proposed 17 qualitative scoring categories which also includes certain defense mechanisms like denial.

Hand test has four major domains i.e. Interpersonal which encompasses affection, dependence, communication, exhibition, direction and aggression. The second major category is Environmental, its sub categories are action, acquisition and passive. The third main domain of mal-adjective category represents neurotic protocol which includes tension, crippled and fear responses. Whereas the fourth main domain of withdrawal category represents psychotic protocol and consists of description, fail and bizarre. (Wagner, 1983)

Qualitative scoring categories have also been described and these include

- $\quad$ Ambivalent: e.g., Could be hitting someone, thumb is in wrong place

- $\quad$ Automatic phrase e.g., She is showing off her ring, I guess that's all I see

- $\quad$ Cylindrical: e.g., Plumber screwing in a pipe

- $\quad$ Denial: e.g., Shaking hand, No that is the wrong hand

- $\quad$ Emotion: e.g., Real happy, just bursting right out with joy

- $\quad$ Gross: e.g., Hand has brass necklace on it

- $\quad$ Hiding: e.g., No one can copy her answer

- $\quad$ Immature: e.g., Patting a dog

- Impotent: e.g., These are too hard for me

- Inanimate: e.g., A beautiful hand like a statue

- Movement: e.g., Like it a folding and unfolding, closing and opening

- $\quad$ Oral: e.g., Has a fork in his hand for eating

- $\quad$ Perplexity: e.g., He, She is puzzled, difficult in generating response

- $\quad$ Sensual: e.g. He is enjoying and playing with his fingers

- $\quad$ Sexual: e.g., Grabbing a women

- $\quad$ Original e.g. Knitting with her fingers

Repetition e.g. previous responses is given again

During modification process of Hand test a total of ten stimulus were initially selected by a panel of professionals, out of which four stimulus were adapted after try out study on a sample of 100 participants (50 normal and 50 psychotic). Stimulus were selected on the basis of their capacity to generate responses in more scoring categories and it is believed that the adapted stimulus are generally used by people of Pakistan as non-verbal communication cue, hence the modified version of Hand test will measure cultural input as claimed by Anastasi (1986) "no single test can be entirely culture free and its results are based on norms of that population which are prone to favor to its inhabitants." It was also believed that new projective techniques should have specific percept which means that stimulus should measure specific aspects of personality rather than overall personality and should have the ability measure some defense mechanisms (Paul Kline, 1986).

(c) The International Journal of Indian Psychology, ISSN 2348-5396 (e)| ISSN: 2349-3429 (p) | 


\section{Qualitative Analysis of Modified Hand Test}

Most of the researches have been carried out on quantitative scoring categories offered by its author and literature is almost silent on qualitative scoring categories of Hand test except the data presented in Hand test manual. It is therefore considered viable to have insight into richness of content based on qualitative categories with the objective to explore certain new areas and dimensions of personality.

\section{METHOD}

\section{Sample}

The sample of this study consisted of 500 participants which was further bifurcated into four major groups i.e. normal which were 350 with equal proportion of males and females, their age ranging from 10 to 80 years with the mean age of 37.01 and (SD) 18.6. The second group consisted of 50 participants i.e. 42 males and 8 females their age ranging from 11 to 46 years with mean age of 20.43 and SD (10.8), these participants had adjustment problems at home as well as at educational institutions as reported by their teachers and administrative staff. The third group consisted of neurotic participants and further divided into three sub group with almost equal number of males and females, in neurotic anxiety their age ranging from 16 to 37 with mean age 34 and SD (9), in neurotic Obsessive Compulsive Disorder, their age ranged from 23 to 30 years with mean age as 36 and SD (9), in neurotic fear their age ranged from 11 to 45 years mean age as 28 and SD (12). They were diagnosed patients of anxiety disorder, obsessive compulsive disorder and phobic disorder. The fourth group consisted of 50 psychotic patients bifurcated into three sub groups with almost equal number of males and females and were diagnosed patients of schizophrenia age ranging from 16 to 57 years with mean age as 31 and SD (11), bipolar age ranging from 21 to 56 years old with mean age as 35 and SD (10) and depression age ranging from 15 to 54 years with mean age as 30 and SD (10), they all were diagnosed by Psychiatrists and Clinical Psychologists.

\section{Procedure}

Purposive sampling technique was used to select participants. The instrument was administered individually in accordance with standardized procedures in Urdu. The scoring booklet was translated into Urdu by a panel of experts. Ethical procedures which includes consent and debriefing were followed. Only psychotic subjects were offered with the example of Hand shake in order to take advantage of testing the limits procedure (Anastasi, 1997) and prompt was also offered only in the beginning but was not followed later on. After an interval of 100 seconds the new card was presented and if response was not offered during this period it was scored as fail. Frequency counts were made in accordance with qualitative scoring categories however scoring categories were omitted where the score was too low. During the content analysis of responses certain new categories were identified and have been added. 


\section{Qualitative Analysis of Modified Hand Test}

\section{RESULTS}

Results have been computed in frequency counts/ percentages along various qualitative scoring categories for normal, maladjusted, neurotic and psychotic groups in the following manner.

Table 1, Data for the Qualitative Scoring Categories, Normal Group $(N=350)$

\begin{tabular}{|c|c|c|c|c|c|c|c|c|c|c|c|c|c|c|c|c|c|c|c|c|c|c|c|c|c|c|c|}
\hline S NO & \multicolumn{1}{|c|}{ Ambivalent } & \multicolumn{2}{|c|}{ Automatic Ph } & \multicolumn{2}{|c|}{ Cylindrical } & \multicolumn{2}{|c|}{ Hiding } & \multicolumn{3}{|c|}{ Immature } & \multicolumn{3}{|c|}{ Impotent } & \multicolumn{3}{|c|}{ Movement } & \multicolumn{3}{|c|}{ Oral } & \multicolumn{2}{|c|}{ Perplexity } \\
\hline & $\mathbf{M}$ & $\mathbf{F}$ & $\mathbf{T}$ & $\mathbf{M}$ & $\mathbf{F}$ & $\mathbf{T}$ & $\mathbf{M}$ & $\mathbf{F}$ & $\mathbf{T}$ & $\mathbf{M}$ & $\mathbf{F}$ & $\mathbf{T}$ & $\mathbf{M}$ & $\mathbf{F}$ & $\mathbf{T}$ & $\mathbf{M}$ & $\mathbf{F}$ & $\mathbf{T}$ & $\mathbf{M}$ & $\mathbf{F}$ & $\mathbf{T}$ & $\mathbf{M}$ & $\mathbf{F}$ & $\mathbf{T}$ & $\mathbf{M}$ & $\mathbf{F}$ & $\mathbf{T}$ \\
\hline 1 & 23 & 18 & 41 & 4 & 0 & 4 & 0 & 0 & 0 & 0 & 0 & 0 & 3 & 5 & 8 & 0 & 0 & 0 & 2 & 1 & 3 & 0 & 0 & 0 & 0 & 1 & 1 \\
\hline 2 & 14 & 10 & 24 & 5 & 1 & 6 & 0 & 0 & 0 & 0 & 0 & 0 & 6 & 6 & 12 & 2 & 4 & 6 & 10 & 9 & 19 & 0 & 0 & 0 & 0 & 1 & 1 \\
\hline 3 & 9 & 8 & 17 & 3 & 0 & 3 & 0 & 0 & 0 & 0 & 0 & 0 & 1 & 1 & 2 & 0 & 0 & 0 & 0 & 0 & 0 & 0 & 0 & 0 & 0 & 0 & 0 \\
\hline 4 & 11 & 8 & 19 & 2 & 2 & 4 & 0 & 0 & 0 & 0 & 0 & 0 & 3 & 2 & 5 & 0 & 0 & 0 & 11 & 8 & 19 & 0 & 0 & 0 & 0 & 1 & 1 \\
\hline 5 & 5 & 8 & 13 & 2 & 1 & 3 & 1 & 0 & 1 & 0 & 0 & 0 & 3 & 3 & 6 & 42 & 55 & 97 & 5 & 4 & 9 & 0 & 0 & 0 & 1 & 0 & 1 \\
\hline 6 & 15 & 18 & 33 & 0 & 1 & 1 & 0 & 0 & 0 & 2 & 6 & 8 & 1 & 1 & 2 & 1 & 1 & 2 & 4 & 2 & 6 & 2 & 0 & 2 & 1 & 0 & 1 \\
\hline 7 & 13 & 9 & 22 & 1 & 0 & 1 & 4 & 6 & 10 & 3 & 0 & 3 & 6 & 4 & 10 & 1 & 1 & 2 & 4 & 2 & 6 & 2 & 3 & 5 & 0 & 0 & 0 \\
\hline 8 & 13 & 10 & 23 & 2 & 2 & 4 & 1 & 0 & 1 & 1 & 2 & 3 & 13 & 7 & 20 & 2 & 2 & 4 & 1 & 2 & 3 & 12 & 11 & 23 & 0 & 0 & 0 \\
\hline 9 & 7 & 7 & 14 & 1 & 2 & 3 & 0 & 0 & 0 & 3 & 1 & 4 & 10 & 9 & 19 & 16 & 17 & 33 & 7 & 5 & 12 & 0 & 0 & 0 & 2 & 0 & 2 \\
\hline 10 & 9 & 6 & 15 & 1 & 1 & 2 & 5 & 4 & 9 & 0 & 0 & 0 & 8 & 5 & 13 & 2 & 3 & 5 & 1 & 0 & 1 & 0 & 0 & 0 & 0 & 0 & 0 \\
\hline 11 & 5 & 3 & 8 & 1 & 0 & 1 & 1 & 0 & 1 & 2 & 4 & 6 & 12 & 9 & 21 & 3 & 4 & 7 & 8 & 2 & 10 & 0 & 1 & 1 & 1 & 0 & 1 \\
\hline 12 & 6 & 5 & 11 & 0 & 1 & 1 & 0 & 0 & 0 & 0 & 0 & 0 & 2 & 2 & 4 & 0 & 1 & 1 & 5 & 1 & 6 & 1 & 2 & 3 & 1 & 0 & 1 \\
\hline 13 & 16 & 5 & 21 & 0 & 0 & 0 & 0 & 0 & 0 & 0 & 0 & 0 & 4 & 0 & 4 & 1 & 0 & 1 & 5 & 1 & 6 & 1 & 2 & 3 & 0 & 1 & 1 \\
\hline 14 & 6 & 7 & 13 & 0 & 1 & 1 & 0 & 0 & 0 & 0 & 0 & 0 & 3 & 2 & 5 & 1 & 2 & 3 & 3 & 0 & 3 & 0 & 0 & 0 & 1 & 1 & 2 \\
\hline Total & 152 & 122 & 274 & 22 & 12 & 34 & 12 & 10 & 22 & 11 & 13 & 24 & 75 & 56 & 131 & 71 & 90 & 161 & 66 & 37 & 103 & 18 & 19 & 37 & 7 & 5 & 12 \\
\hline
\end{tabular}

\begin{tabular}{|c|c|c|c|c|c|c|c|c|c|c|c|c|c|c|c|c|c|c|c|c|}
\hline \multicolumn{3}{|c|}{ Sensual } & \multicolumn{3}{|c|}{ Repetition } & \multicolumn{3}{|c|}{ Introjections } & \multicolumn{3}{|c|}{ Compensation } & \multicolumn{3}{|c|}{ Oppositional } & \multicolumn{3}{|c|}{ Religiosity } & \multicolumn{3}{|c|}{ Original } \\
\hline M & $\mathbf{F}$ & $\mathbf{T}$ & $\mathbf{M}$ & $F$ & $\mathbf{T}$ & M & $\mathbf{F}$ & $\mathbf{T}$ & M & $\mathbf{F}$ & $\mathbf{T}$ & M & $\mathbf{F}$ & $\mathbf{T}$ & M & $\mathbf{F}$ & $\mathbf{T}$ & M & $\mathbf{F}$ & $\mathbf{T}$ \\
\hline 1 & 0 & 1 & 2 & 0 & 2 & 2 & 2 & 4 & 0 & 0 & 0 & 0 & 0 & 0 & 2 & 2 & 4 & 0 & 0 & 0 \\
\hline 0 & 0 & 0 & 3 & 5 & 8 & 1 & 0 & 1 & 1 & 0 & 1 & 2 & 0 & 2 & 0 & 1 & 1 & 3 & 0 & 3 \\
\hline 0 & 0 & 0 & 2 & 5 & 7 & 0 & 1 & 1 & 0 & 0 & 0 & 0 & 0 & 0 & 5 & 3 & 8 & 0 & 0 & 0 \\
\hline 1 & 0 & 1 & 3 & 3 & 6 & 1 & 2 & 3 & 0 & 0 & 0 & 2 & 0 & 2 & 0 & 2 & 2 & 2 & 0 & 2 \\
\hline 3 & 0 & 3 & 8 & 5 & 13 & 1 & 0 & 1 & 1 & 0 & 1 & 0 & 0 & 0 & 2 & 2 & 4 & 3 & 6 & 9 \\
\hline 0 & 0 & 0 & 7 & 4 & 11 & 0 & 0 & 0 & 0 & 0 & 0 & 0 & 1 & 1 & 1 & 1 & 2 & 2 & 1 & 3 \\
\hline 0 & 0 & 0 & 0 & 5 & 5 & 0 & 0 & 0 & 4 & 1 & 5 & 1 & 0 & 1 & 4 & 3 & 7 & 1 & 3 & 4 \\
\hline 0 & 0 & 0 & 1 & 4 & 5 & 0 & 0 & 0 & 1 & 1 & 2 & 1 & 0 & 1 & 3 & 2 & 5 & 3 & 0 & 3 \\
\hline 1 & 1 & 2 & 3 & 8 & 11 & 0 & 0 & 0 & 1 & 1 & 2 & 1 & 0 & 1 & 5 & 11 & 16 & 0 & 2 & 2 \\
\hline 1 & 0 & 1 & 1 & 8 & 9 & 1 & 0 & 0 & 0 & 1 & 1 & 0 & 0 & 0 & 34 & 22 & 56 & 0 & 1 & 1 \\
\hline 0 & 0 & 0 & 5 & 13 & 18 & 0 & 0 & 0 & 0 & 0 & 0 & 0 & 0 & 0 & 10 & 5 & 15 & 5 & 3 & 8 \\
\hline 2 & 1 & 3 & 10 & 6 & 16 & 0 & 0 & 0 & 0 & 0 & 0 & 0 & 0 & 0 & 4 & 2 & 6 & 4 & 2 & 6 \\
\hline 0 & 0 & 0 & 4 & 4 & 8 & 0 & 0 & 0 & 0 & 0 & 0 & 0 & 0 & 0 & 33 & 29 & 62 & 9 & 1 & 10 \\
\hline 0 & 0 & 0 & 5 & 6 & 11 & 1 & 1 & 1 & 2 & 0 & 2 & 0 & 0 & 0 & 20 & 15 & 35 & 3 & 2 & 5 \\
\hline 9 & 2 & 11 & 54 & 76 & 130 & 7 & 6 & 11 & 10 & 4 & 14 & 7 & 1 & 8 & 123 & 100 & 223 & 35 & 21 & 56 \\
\hline & & & & & & & & & & & & & & & & & & & & \\
\hline
\end{tabular}

Qualitative analysis was carried out along with 17 qualitative categories, after going through content analysis of the responses of Pakistani sample of Normal, maladjusted, neurotic and psychotic, certain new qualitative aspects are explored and incorporated in the present study. These are related to Pakistani culture e.g., Religiosity and some defense mechanisms like introjections, opposition, compensation, projection, need for affiliation, need for achievement

(c) The International Journal of Indian Psychology, ISSN 2348-5396 (e) | ISSN: 2349-3429 (p) | 154 


\section{Qualitative Analysis of Modified Hand Test}

and altruism, etc. However only 16 categories were retained, where the input was in reasonable quantity. The qualitative data provides insight into the dynamics of mind-set, exposure and experiences of respondents, e.g., a peasant who had been involved in his role of farmer throughout life gave responses in accordance with his experiences and when sequence analysis was carried out(a traditional method), responses revealed around his previous way of handling the environment and pattern of dealing with others, few responses include bringing grass for animals, preparing land for dipping seeds etc. A soldier and a mechanic just cannot go out while responding from their own way of life e.g. mechanic responded on card 12 that asking for a certain number bolt. Difference in responses were also oriented from Normality to Maladjustment, neurotic to psychotic groups, their brief description is appended below.

274 responses came out in ambivalent category of modified Hand test. They gave responses having origin of indecisiveness or contradictory in nature, e.g., Different two responses of a person on card one also indicate bipolar pattern of the invidual. 'Salam le raha hai (shaking hand) Thappar maar raha hai (slapping)". Gender differences were also found e.g., in the ambivalent category females were constituting $45 \%$ and males were $55 \%$. These responses were not equally distributed along with various stimulus e.g., the highest constellation of responses in ambivalent category was on stimulus one followed by stimulus two, six and thirteen respectively. The second highest score obtained by normal population came out in Religiosity i.e.223. Here the major share was contributed from newly introduced stimulus 10,13 and 14 respectively. The third main category remained impotent i.e.161, here the inverse ratio was found between male and female and female exceeded with $55 \%$ to male, $45 \%$. Here the major portion of responses was contributed from stimulus five. It is to highlight in quantitative data the responses on this stimulus was interpreted as crippled, this has reflected the stimulus constancy (pull of stimulus) however is matched with inner dynamics of personality. Almost equal number of responses were obtained by immature and repetition categories i.e. 131 and 130. Here male remained relatively more immature constituting 57\% whereas females were $43 \%$. The contribution of responses came out from stimulus 11 (newly introduced stimulus), eight and nine. Females were more in number and constitute 58\% as compared to males with $42 \%$. A total of 103 responses were found in movement category, out of which $65 \%$ were male and $35 \%$ were female. The major contribution of responses were from stimulus two, four, nine and eleven (Newly introduced stimulus) respectively. The lowest score was obtained in opposition i.e. eight followed by introjections and sensual i.e.11 and compensation i.e. 14. 


\section{Qualitative Analysis of Modified Hand Test}

Table 2, Data for the Qualitative Scoring Categories, Maladjustment Group ( $N=50)$

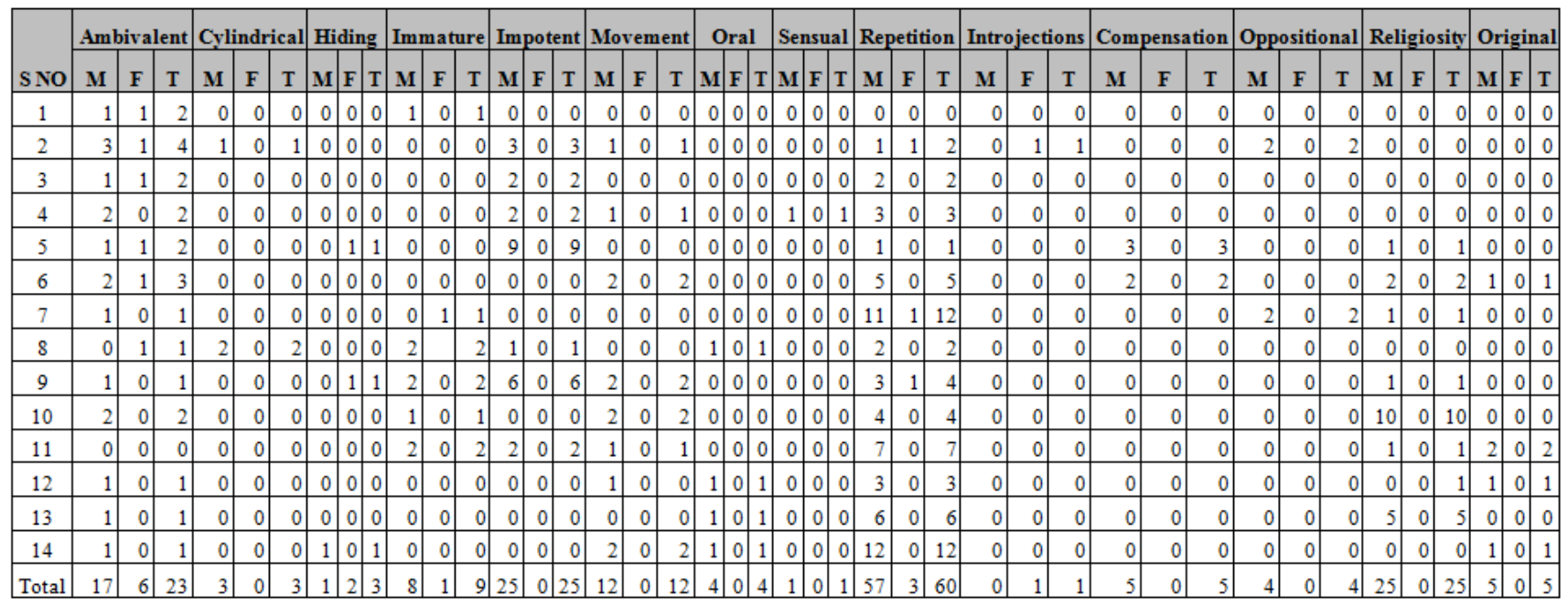

It is pertinent to mention here that number of categories were reduced to 14. The highest 60 number of responses were found in repetition by maladjusted group, followed by 25 number of responses each in immature and religiosity i.e. 25 and 23 number of responses were found in ambivalent category. Lower number of responses were found in introjection, sensual, ambivalent, cylindrical, oppositional, and oral and movement.

Table 3, Data for the Qualitative Scoring Categories, Neurotic Group (N=50)

\begin{tabular}{|c|c|c|c|c|c|c|c|c|c|c|c|c|c|c|c|c|c|c|c|c|c|c|c|c|c|c|c|c|c|c|c|c|c|c|c|c|c|c|c|c|c|}
\hline \multirow{2}{*}{$\begin{array}{c}\mathrm{S} \\
\text { No }\end{array}$} & \multicolumn{3}{|c|}{ Ambivalent } & \multicolumn{3}{|c|}{ Cylindrical } & \multicolumn{2}{|c|}{ Hiding } & \multicolumn{3}{|c|}{ Immature } & \multicolumn{2}{|c|}{ Impotent } & \multicolumn{4}{|c|}{ Movement } & \multicolumn{2}{|c|}{ Oral } & \multicolumn{3}{|c|}{ Perplexity } & \multicolumn{2}{|c|}{ Sensual } & \multicolumn{3}{|c|}{ Repetition } & \multicolumn{3}{|c|}{ Introjection } & \multicolumn{3}{|c|}{ Compensation } & \multicolumn{3}{|c|}{ Oppositional } & \multicolumn{3}{|c|}{ Religiosity } & \multicolumn{2}{|c|}{ Original } \\
\hline & M & $F$ & $\mathrm{~T}$ & M & $F$ & $\mathrm{~T}$ & M F & & $\mathbf{M}$ & $\mathbf{F}$ & $\mathrm{T}$ & M $\mathbf{I}$ & E $\mathrm{I}$ & 5 & $\mathrm{M}$. & & T 1 & $\mathrm{M} F$ & $\mathrm{~T}$ & M 1 & $\mathrm{~F}$ & & $\mathrm{~F}$ & & M. & $\mathbf{F}{ }_{1}$ & $\mathrm{~T} \quad 1$ & M & $\mathbf{F}$ & $\mathrm{T}$ & M & $\mathbf{F}$ & $\mathrm{T}$ & M & $\mathbf{F}$ & $\mathrm{T}$ & M & $\mathbf{F}$ & $\mathrm{T} 1$ & $\mathrm{M} F$ & $\mathrm{~T}$ \\
\hline 1 & & & 2 & & & & & $a_{0}$ & & & 0 & & $\underline{0}$ & & & & 0 & 0.0 & & & & & 00 & 0 & 0 & 0 & & & & & & & & & & & 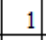 & & & & \\
\hline & & & 3 & & & & \begin{tabular}{l|l}
0 & 0 \\
\end{tabular} & 0 & & & & 0 & 0 & & & & 0 & $\begin{array}{lll}0 & 0 \\
\end{array}$ & 0 & & & & 00 & 0 & & & & & & & & & & & & & & & & 7 & \\
\hline 3 & & & 2 & & & 0 & \begin{tabular}{l|l}
0 & 0 \\
\end{tabular} & 0 & & & of & 0 & 0 & 0 & & & 0 & $\begin{array}{lll}0 & 0 \\
\end{array}$ & 0 & & 0 & & $\begin{array}{lll}0 & 0 \\
\end{array}$ & 0 & 0 & 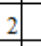 & & 4 & - & 0 & & & & & & & 2 & 0 & 2 & 00 & \\
\hline 4 & & 1 & 2 & 0 & 0 & 0 & 00 & 0 & & & 0 & 0 & 0 & $\underline{0}$ & & & 0 & $\begin{array}{lll}0 & 0 \\
\end{array}$ & 0 & 0 & 0 & - & 00 & 0 & 1 & 2 & 3 & 0 & 4 & 0 & & & & & & & 0 & & & $\begin{array}{ll}0 & 1 \\
\end{array}$ & \\
\hline & & 2 & 3 & 0 & & 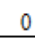 & 00 & 0 & & & 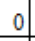 & & $\begin{array}{lll}5 & 1 \\
\end{array}$ & & & & 1 & 00 & 0 & & 0 & & 00 & 0 & 2 & 4 & & 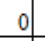 & 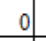 & & & 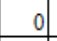 & & & & & 4 & 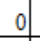 & 0 & $\begin{array}{ll}0 & 1 \\
\end{array}$ & \\
\hline 6 & 1 & 2 & 3 & 0 & $\underline{0}$ & 0 & $\begin{array}{lll}0 & 1 \\
\end{array}$ & 1 & t & ( & 0 & 1 & 0 & 1 & t & 1 & 1 & $\begin{array}{lll}0 & 0 \\
\end{array}$ & 0 & 0 & 1 & 1 & 00 & 0 & 1 & 5 & 6 & 1 & 0 & 1 & 0 & 0 & 1 & 0 & & & 1 & 1 & 2 & 012 & \\
\hline & & 1 & 5 & 0 & & 0 & $\begin{array}{lll}0 & 1 \\
\end{array}$ & + & & & & - & 0 & & & & 2 & $\begin{array}{lll}0 & 0 \\
\end{array}$ & 0 & - & 0 & & 0 & 0 & & & & 4 & & & & & & & & & 2 & & & 0 & \\
\hline 8 & & & 3 & 1 & 0 & 1 & 00 & 0 & 0 & 0 & 0 & 2 & 1 & 3 & & 1 & 1 & 12 & 3 & 0 & 3 & 3 & 00 & 0 & 1 & 0 & 1 & 0 & 0 & 0 & & 0 & & 0 & & & 1 & 0 & 1 & \begin{tabular}{l|l}
0 & 1 \\
0
\end{tabular} & \\
\hline 9 & & 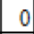 & 2 & 0 & 1 & 1 & 00 & & & & 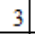 & 7 & 1 & 8 & & & 3 & $\begin{array}{lll}0 & 0 \\
\end{array}$ & & 0 & & & $\begin{array}{lll}0 & 1 \\
\end{array}$ & 1 & 2 & & & 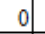 & 0 & 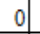 & & & & & & & 1 & & & $\begin{array}{lll}0 & 0\end{array}$ & \\
\hline 10 & 4 & 0 & 1 & 0 & 1 & 1 & 00 & & 0 & ( & 0 & 4. & 1 & 5 & 0 & & 0 & $\begin{array}{lll}0 & 1 \\
\end{array}$ & & 1 & 0 & & $\begin{array}{lll}0 & 0 \\
\end{array}$ & 0 & 1 & 4 & 5 & 0 & 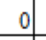 & 0 & ㄴ & 의 & & 의 & 0 & & 4 & 2 & & $\begin{array}{lll}0 & 1 \\
\end{array}$ & \\
\hline 11. & & 0 & 2 & 0 & 0 & 0 & 00 & & 0 & & 1 & 0 & 0 & 0 & & & 3 & 00 & & 0 & 0 & 0 & $\begin{array}{lll}0 & 0 \\
\end{array}$ & 0 & 3 & 5 & 8 & 0 & 0 & 0 & 0 & 4 & & 0 & & & 1 & 1 & & $\begin{array}{lll}0 & 0 \\
\end{array}$ & \\
\hline 12 & & 0 & 1 & 0 & 0 & 0 & 00 & & 0 의 & 0 & 0 & \begin{tabular}{l|l}
0 \\
\end{tabular} & 0 & 0 & $t$ & $\underline{0}$ & 0 & 10 & & 1 & 0 & 1 & 10 & 1 & 2 & 4 & 3 & 1 & 0 & 1 & 4 & 0 & & 0 & 0 & 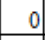 & 1 & 2 & 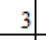 & 10 & \\
\hline & & 0 & & 0 & 0 & 0 & \begin{tabular}{l|l}
0 & 0 \\
\end{tabular} & & 0 & 0 & 0 & 0 & 0 & 0 & 0 & & 1 & $\begin{array}{lll}10 \\
\end{array}$ & & 0 & 0 & 0 & 00 & 0 & 0 & 4 & 4 & 1 & 0 & 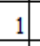 & 0 & 0 & & 0 & 0 & 0 & 2 & 2 & 4 & $\begin{array}{lll}0 & 1 \\
\end{array}$ & \\
\hline 14 & 4 & 2 & 4 & 0 & 0 & 0 & $\begin{array}{lll}0 & 1 \\
\end{array}$ & & 1 & 0 & 1 & 0 & 0 & 0 & 0 & $\underline{0}$ & 0 & $\begin{array}{lll}0 & 0 \\
\end{array}$ & 0 & 0 & 0 & 0 & 00 & 0 & 0 & & 4 & 0 & 0 & 0 & 0 & 0 & & 1 & 0 & 1 & 2 & 0 & 2 & 2 & \\
\hline & 20 & 13 & 33 & 1 & 2 & 3 & $\begin{array}{lll}0 & 3 \\
\end{array}$ & & 3 & - & 7 & 20 & \begin{tabular}{l|l}
8 & 28
\end{tabular} & & & & 13 & $\begin{array}{lll}3 & 3 \\
\end{array}$ & 6 & - & 6]. & 1 & ل & 2 & 15 & $34]$ & 49 & & $a_{0}$ & & & & & & & & 18 & & 27 & & 1 \\
\hline
\end{tabular}

It is to highlight here that number of categories were reduced to 15 . Highest 49 number of responses were found again in repetition category by neurotic group which include $69 \%$ female and $31 \%$ male followed by ambivalent 33 in number responses $61 \%$ male and 39\% female, impotent 28 number of responses, 71\% male and $29 \%$ female and religiosity 27 number of responses, $67 \%$ male and 33\% female respectively. Lower number of responses were obtained 


\section{Qualitative Analysis of Modified Hand Test}

by neurotic group in hiding, cylindrical, oral and introjection with three each responses and sensual and compensation with two responses in each category.

\section{Table 4, Data for the Qualitative Scoring Categories, Psychotic Group ( $N=50)$}

\begin{tabular}{|c|c|c|c|c|c|c|c|c|c|c|c|c|c|c|c|c|c|c|c|c|c|c|c|c|c|c|c|c|c|c|c|c|c|c|c|c|c|}
\hline \multirow[b]{2}{*}{ S No } & \multicolumn{3}{|c|}{ Ambivalent } & \multicolumn{3}{|c|}{ Cylindrical } & \multicolumn{3}{|c|}{ Immature } & \multicolumn{3}{|c|}{ Impotent } & \multicolumn{3}{|c|}{ Movement } & \multicolumn{2}{|c|}{ Oral } & \multicolumn{3}{|c|}{ Perplexity } & \multicolumn{2}{|c|}{ Sensual } & \multicolumn{3}{|c|}{ Repetition } & \multicolumn{3}{|c|}{ Introjection } & \multicolumn{3}{|c|}{ Compensation } & \multicolumn{3}{|c|}{ Oppositional } & \multicolumn{3}{|c|}{ Religiosity } \\
\hline & $\mathbf{M}$ & $\mathbf{F}$ & $\mathrm{T}$ & M & $\mathbf{F}$ & $\mathrm{T}$ & M & $\mathbf{F}$ & $\mathrm{T}$ & $\mathbf{M}$ & F & $\mathrm{T}$ & M & $F$ & $\mathrm{~T}$ & $M F$ & $\mathrm{~T}$ & $\mathbf{M}$ & $\mathbf{F}$ & $\mathrm{T}$ & $M I$ & $\mathrm{~T}$ & M & $F$ & $\mathrm{~T}$ & $\mathbf{M}$ & $\mathbf{F}$ & $\mathrm{T}$ & M & F & $\mathrm{T}$ & $\mathbf{M}$ & F & $\mathrm{T}$ & M & F & $\mathrm{T}$ \\
\hline 1 & 3 & 1 & 4 & 0 & 0 & 0 & 0 & 0 & 0 & 3 & 0 & 3 & 0 & \begin{tabular}{|l|}
0 \\
\end{tabular} & 0 & \begin{tabular}{l|l}
0 & 0 \\
\end{tabular} & 0 & 0 & 0 & 0 & \begin{tabular}{l|l}
0 & 0 \\
\end{tabular} & 0 & 1 & 0 & 1 & 0 & 0 & 0 & 0 & 0 & 0 & 0 & 0 & 0 & 0 & 0 & 0 \\
\hline 2 & 0 & 2 & 2 & 0 & 0 & 0 & 0 & 0 & 0 & 1 & 0 & 1 & 0 & 0 & 0 & \begin{tabular}{l|l}
0 & 0
\end{tabular} & 0 & 0 & 0 & 0 & $\begin{array}{lll}0 & 0\end{array}$ & 0 & 3 & 0 & 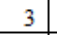 & 0 & 0 & $u$ & 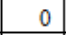 & 0 & 0 & 1 & 0 & 1 & 0 & 1 & 1 \\
\hline 3 & 0 & 1 & 1 & 0 & 0 & 0 & 0 & 0 & 0 & 0 & 0 & 0 & 0 & 0 & 0 & \begin{tabular}{l|l}
0 & 0 \\
\end{tabular} & 0 & 0 & 0 & 0 & \begin{tabular}{l|l}
0 & 1
\end{tabular} & \begin{tabular}{l|l}
1 & 1 \\
\end{tabular} & 4 & 3 & 7 & 0 & 0 & 0 & 0 & 0 & 0 & 0 & 0 & 0 & 3 & 2 & 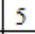 \\
\hline 4 & 0 & 1 & 1 & 0 & 0 & 0 & 0 & 0 & 0 & 0 & 1 & 0 & 0 & 0 & 0 & 0.0 & 0 & 1 & 0 & 1 & $\begin{array}{lll}0 & 0\end{array}$ & 0 & 4 & 3 & 7 & 0 & 0 & 0 & 1 & 0 & 1 & 1 & 0 & 1 & 3 & 0 & 3 \\
\hline 5 & 1 & 1 & 2 & 0 & 0 & 0 & 0 & 0 & 0 & 4 & 4 & 8 & 1 & 0 & 1 & 0 & 0 & 3 & 0 & 3 & 00 & 0 & 7 & 5 & 12 & 0 & 0 & 0 & 1 & 0 & 1 & 1 & 0 & 1 & 2 & 0 & 2 \\
\hline 6 & 4 & 5 & 9 & 0 & 0 & 0 & 0 & 0 & 0 & 2 & 0 & 2 & 0 & 0 & 0 & \begin{tabular}{l|l}
0 & 0
\end{tabular} & 0 & 1 & 0 & 1 & $\begin{array}{lll}0 & 0\end{array}$ & 0 & 7 & 6 & 13 & 1 & 0 & 1 & 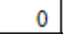 & 0 & 0 & 1 & 0 & 1 & 2 & 0 & 2 \\
\hline 7 & 3 & 3 & 6 & 0 & 0 & 0 & 0 & 1 & 1 & 1 & 1 & 2 & 1 & 0 & 1 & \begin{tabular}{l|l}
0 & 0 \\
\end{tabular} & 0 & 0 & 0 & 0 & $\begin{array}{lll}0 & 0 \\
\end{array}$ & 0 & 5 & 4 & 9 & 0 & 0 & 0 & 0 & 0 & 0 & 0 & 1 & 1 & 1 & 0 & 1 \\
\hline 8 & 2 & 6 & 8 & 2 & 0 & 2 & 5 & 3 & 8 & 3 & 0 & 3 & 0 & 0 & 0 & 32 & 5 & 0 & 0 & 0 & 00 & 0 & 5 & 6 & 11 & 8 & 0 & 8 & 0 & 0 & 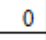 & 0 & 0 & 0 & 1 & 0 & 1 \\
\hline 9 & 0 & 1 & 0 & 1 & 0 & 0 & 0 & 0 & 0 & 5 & 2 & 7 & 1 & 0 & 1 & \begin{tabular}{l|l}
0 & 0 \\
\end{tabular} & 0 & 1 & 0 & 1 & $\begin{array}{lll}0 & 0 \\
\end{array}$ & $\begin{array}{l}0 \\
0\end{array}$ & 5 & 5 & 10 & 0 & 2 & 2 & 0 & 0 & 0 & 0 & 0 & 0 & 1 & 1 & 2 \\
\hline 10 & 2 & 1 & 3 & 0 & 0 & 0 & 0 & 1 & 1 & 2 & 1 & 3 & 0 & 0 & 0 & $\begin{array}{lll}0 & 0 \\
\end{array}$ & 0 & 0 & 0 & 0 & \begin{tabular}{l|l}
1 & 1 \\
\end{tabular} & 12 & 5 & 5 & 10 & 5 & 3 & 8 & 0 & 0 & 0 & 0 & 0 & 0 & 0 & 0 & 0 \\
\hline 11 & 3 & 1 & 4 & 2 & 1 & 3 & 2 & 0 & 2 & 0 & 1 & 1 & 0 & 0 & 0 & \begin{tabular}{l|l}
0 & 1 \\
\end{tabular} & 1 & 2 & 0 & 2 & $\begin{array}{lll}0 & 0\end{array}$ & 0 & 6 & 6 & 12 & 2 & 1 & 3 & 0 & 0 & 0 & 0 & 0 & 0 & 1 & 0 & 1 \\
\hline 12 & 2 & 2 & 4 & 0 & 0 & 0 & 0 & 1 & 1 & 0 & 0 & 0 & 0 & 0 & 0 & \begin{tabular}{l|l}
1 & 1 \\
\end{tabular} & 2 & 0 & 0 & 0 & 10 & 1 & 8 & 9 & 17 & 0 & 0 & 0 & 0 & 0 & 0 & 0 & 0 & 0 & 1 & 0 & 1 \\
\hline 13 & 1 & 0 & 1 & 0 & 0 & 0 & 0 & 0 & 0 & 0 & 0 & 0 & 0 & 0 & 0 & \begin{tabular}{l|l}
1 & 1 \\
\end{tabular} & 2 & 0 & 0 & 0 & \begin{tabular}{l|l}
0 & 1
\end{tabular} & \begin{tabular}{l|l}
1 & 1 \\
\end{tabular} & 5 & 5 & 10 & 0 & 0 & 0 & 0 & 0 & 0 & 0 & 0 & 0 & 5 & & 10 \\
\hline 14 & 1 & 1 & 2 & 1 & 0 & 1 & 0 & 1 & 1 & 0 & 0 & 0 & 0 & 1 & 1 & \begin{tabular}{l|l}
1 & 0
\end{tabular} & 1 & 1 & 0 & 1 & $\begin{array}{lll}0 & 0\end{array}$ & 0 & 6 & 5 & 11 & 1 & 0 & 1 & 2 & 4 & 6 & 0 & 0 & 0 & 0 & 0 & 0 \\
\hline Total & 22 & 26 & 48 & 6 & 1 & 7 & 7 & & 14 & 21 & & & 3 & 1 & 4 & \begin{tabular}{l|l}
6 & 5 \\
\end{tabular} & 11 & 9 & 0 & 9 & 23 & 3. & & & 133 & 17 & 6 & 23 & 4 & 4 & 8 & 4 & 1 & 5 & 20 & & 29 \\
\hline
\end{tabular}

The highest 133 number of responses were found in repetition by psychotic group constituting $53 \%$ males and $47 \%$ females followed by 48 number of responses in ambivalent category consisting 46\% males and $54 \%$ females, 31 number of responses were found in impotent category out of which $68 \%$ were male and 32\% were female and 29 number of responses were found in religiosity category where $72 \%$ were male and $28 \%$ were female.

\section{DISCUSSION}

\section{(Normal group)}

Qualitative analysis of stimulus of modified Hand test revealed that there were difference of gender constituting percentages of responses though the difference were not much. E.g. more immature were male than females, similarly more number of responses were given by male in movement category, they also scored more in religiosity. New dimensions e.g. need achievement, need for affiliation, altruism were explored and persons belonging to health professions e.g. doctors projected altruism. Teachers demonstrated direction, orientation.

Contribution of new stimulus were also identified and reflected cultural impact, more number of responses were given in the context of religion on stimulus 10 and 13 . There were some contribution in this regard of stimulus three and the same has been identified for the first time in the present study. Another category of oppositional was also identified, such persons tend to adapt an oppositional stance which is an indication of their inability to deal effectively with the outer environment especially with authority figure. This also revealed from their brief case histories during the post- test probe such responses were identified on stimulus four which 


\section{Qualitative Analysis of Modified Hand Test}

contains father percept and stimulus seven. It is pertinent to mention that there was hardly any response found in sexual category however few responses were found in the sensual category. This trend is otherwise representation of normal individuals and characteristics of people of this region.

\section{(Maladjusted Group)}

Pattern and sequence of responses have been changed here as highest scoring category which is characteristic of this group remained repetition and an indication of feeble mindedness, lack of intellect and flexibility. However this pattern of repetition and religiosity may also be an indication of covering up mechanism by this group, they have also given more number of responses in description category in quantitative data where they only described the position of stimulus instead of responding about the action. It is to highlight when maximum number of responses came out as repetition, the quality of responses is also compromised.

\section{(Neurotic Group)}

The similar pattern have been found in terms of manner of responses of these two groups of maladjustment and neurotics. Both have higher repetition responses. Here again, male supersedes females in certain category like religiosity, impotence etc. Hiding was also observed from both males and females. This hiding has been found in addition to description responses, it is to highlight that hiding was generally found on stimulus six where the pull is for aggression response and this is an indication of effort to control their id impulses of aggression.

\section{(Psychotic Group)}

They also demonstrated the same pattern of repetition as was found in maladjusted and neurotic group, however it is interesting to note that in spite their inability to deal effectively and meaningfully with their immediate environment they also gave responses in religious context to somewhat reasonable proportion and it appeared that they tried to get some support of religion. The same can be interpreted as a covering mechanism for their id impulses of sexual and aggression. This perhaps was also observed during the process of test administration and probe. It is to note that this group gave more number of responses in ambivalent category which depicted their inability to discriminate external stimuli with clarity. Defence mechanism of introjection appeared more frequently. This reflects their ego centric approach towards life and is a characteristic of this group.

\section{LIMITATIONS}

The newly introduced stimulus have cultural reference and it was expected that these stimulus are representation of responses in terms of nonverbal cues by the people of this region. Result would have been more conclusive if the modified Hand test was administered to population of other cultures and countries. 


\section{Qualitative Analysis of Modified Hand Test}

\section{IMPLICATIONS}

As cited above most of the studies previously conducted on this instrument are based on quantitative scoring categories, the present study is likely to pave the way to carry out qualitative studies, hence it will provide more insight in understanding dynamics of human functioning. Recommendation

In future studies qualitative analysis of modified Hand test may be carried out with divergent sample and with different cultures. It is also recommended that other qualitative analysis may be incorporated like Interpretative phenomenological responses.

\section{CONCLUSION}

Qualitative and sequence analysis of data has provided interesting results and each group of sample gave responses in different qualitative categories which are considered typical of them, it provides insight about personality integration and configuration of normal, maladjusted, neurotic and psychotic groups.

\section{Acknowledgments}

The author appreciates all those who participated in the study and helped to facilitate the research process.

\section{Conflict of Interests}

The author declared no conflict of interests.

\section{REFERENCES}

Anastasi, A. (1997). Psychology, psychologists, and psychological testing. American Psychologist, 22 (4), 297.

Kline, P. (1986). A handbook of test construction: Introduction to psychometric design. Methuen.

Riaz, M. N. (2008). Test Construction: Development and Standardization of Psychological Tests in Pakistan. Islamabad: HEC.

Wagner, E.E., Maloney, P., \&Wilson, D.G. (1981). Split half and test retest reliability for pathological samples. Journal of Clinical Psychology, 37, 589-592.

Wendler, C. L. W., \& Zachary, R. A. (1983). Reliability of scoring categories on a projective test. In Paper presented at the meeting of the Western Psychological Association.

Zehra, F. (1989). Inter scorer reliability of Hand Test in Pakistan. Pakistan Journal of Psychological Research, 4, 43-46.

How to cite this article: Anjum F (2017), Qualitative Analysis of Modified Hand Test, International Journal of Indian Psychology, Volume 4, Issue 2, No. 95, ISSN:2348-5396 (e), ISSN:2349-3429 (p), DIP:18.01.176/20170402, ISBN:978-1-365-84231-3

(C) The International Journal of Indian Psychology, ISSN 2348-5396 (e)| ISSN: 2349-3429 (p) | 159 